\title{
Schizandra chinensis Alkaloids Inhibit Lipopolysaccharide-Induced Inflammatory Responses in BV2 Microglial Cells
}

\author{
Min Sik ChOI ${ }^{1, \#, ~ K y u n g ~ J a ~ K w o N ~}{ }^{2, \#}$, Se Jin JeON ${ }^{1}$, Hyo Sang Go ${ }^{1}$, Ki Chan KIM ${ }^{1}$, Jae Ryun Ryu ${ }^{1}$, Jongmin LeE ${ }^{2}$, \\ Seol-Heui HAN ${ }^{2}$, Jae Hoon $\mathrm{CHEONG}^{3}$, Jong Hoon RYu ${ }^{4}$, KiHwan BAE ${ }^{5}$, Chan Young SHIN ${ }^{2}$, and Kwang Ho Ko ${ }^{1, *}$ \\ ${ }^{1}$ Department of Pharmacology, College of Pharmacy and Research Institute of Pharmaceutical Sciences, \\ Seoul National University, Seoul 151-742, ${ }^{2}$ Center for Geriatric Neuroscience Research, IBST, and School of Medicine, \\ Konkuk University, Seoul 143-701, ${ }^{3}$ Department of Pharmacy, Sahmyook University, Seoul 139-742, \\ ${ }^{4}$ Department of Oriental Pharmaceutical Science, College of Pharmacy, Kyung Hee University, Seoul 130-701, \\ ${ }^{4}$ College of Pharmacy, Chungnam University, Daejun 301-721, Republic of Korea
}

(Received December 9, 2008; Revised January 9, 2009; Accepted January 12, 2009)

\begin{abstract}
Schizandra chinensis (S. chinensis) exhibits a harmless, 'adaptogen-type' effect leading to improvements in mental performance and learning efficacy in brain. Activated microglia contributes to neuronal injury by releasing neurotoxic products, which make it important to regulate microglial activation to prevent further cytological as well as functional brain damage. However, the effect of $S$. chinensis on microglial activation has not been examined yet. We have investigated the effects of four compounds (Gomisin A, Gomisin N, Schizandrin and Schizandrol A) from S. chinensis on lipopolysaccharide (LPS)induced microglial activation. In this study, BV2 microglial cells were activated with LPS and the microglial activation was assessed by up-regulation of activation markers such as nitric oxide (NO), reactive oxygen species (ROS), and matrix metalloproteinase-9 (MMP-9). The results showed that all four compounds significantly reduced the intracellular level of ROS, the release of NO and MMP-9 as well as LPS-induced phosphorylation of ERK1/2. These results strongly suggested that $S$. chinensis may be useful to modulate inflammation-mediated brain damage by regulating microglial activation.
\end{abstract}

Keywords: Schizandra chinensis, Microglia, Activation, Nitric oxide (NO), Reactive oxygen species (ROS), Matrix metalloproteinase-9 (MMP-9)

\section{INTRODUCTION}

Unlike the old belief that brain is an "immune previleged organ", recent experimental results suggested that the inflammation in the central nervous system (CNS), i.e. neuroinflammation, is linked to neurodegenerative diseases, such as Alzheimer's disease (AD) and Parkinson's disease (PD) (Hensley et al., 1995; McGeer and McGeer, 2004a). In this process, microglial activation plays a pivotal role in the regulation of neuroinflammatory phenotypes. For example, highly activated microglial cells have been found in the substantia nigra of PD patients (Banati et al., 1998) as well as in the vicinity or within the amyloid-beta plaques of

\footnotetext{
${ }^{*}$ Corresponding author

Tel: +82-2-880-7848 Fax: +82-2-885-8211

E-mail:khk123@snu.ac.kr

\#These authors equally contributed to this work.
}

Alzheimer's disease patients (Bornemann et al., 2001). Activated microglial cells generate high levels of reactive oxygen species (ROS), nitric oxide (NO), and matrix metalloproteinase-9 (MMP-9) (Darley-Usmar et al., 1995; McGeer and McGeer, 2004b; del Zoppo et al., 2007).

The intracellular reduction-oxidation state is important in maintaining homeostasis and proper cellular functions. However, when this balance is lost, excessive ROS production induces oxidative stress, which contributes to cellular damage through the modulation of factors such as $\mathrm{NF}-\mathrm{kB}$ and AP-1 that regulate gene expression (Pham et al., 2004; Gloire et al., 2006).

Due to its cytotoxic activity, nitric oxide (NO) has also been implicated in a large number of pathological processes (Bruhwyler et al., 1993). The cytotoxicity of NO is increased by reaction with superoxide anion $\left(\mathrm{O}_{2}^{-}\right)$to form the highly reactive peroxynitrite $\left(\mathrm{ONOO}^{-}\right)$(Beckman et al., 1990). Several researchers including us have reported that 
NO from cytokine-stimulated microglia or astrocytes potentiated NMDA-mediated or glucose deprivation-induced neural/glial toxicity (Hewett et al., 1994; Choi and Kim, 1998).

Matrix metalloproteinases (MMPs) are zinc-dependent proteolytic enzymes that are involved in the remodeling of the extracellular matrix $(\mathrm{ECM})$ in a variety of physiological and pathological processes (Yong et al., 1998). Normally, there is a careful balance between cell division, matrix synthesis and matrix degradation, which is under the control of cytokines, growth factors and cell matrix interactions. But under pathological conditions, this balance is altered (Yu et al., 1997). The role of extracellular proteolysis in inflammatory demyelination, originally hypothesized as a mechanism for myelin degradation, is increasingly recognized as a pathogenetic step and as a target for therapy in human demyelinating diseases (Cuzner and Opdenakker, 1999).

Mitogen-activated protein kinase (MAPK) pathways are important signal transducing cascades that transmit extracellular signals to an appropriate response (Chang and Karin, 2001). The extracellular signal-regulated kinases 1 and 2 (ERK1/2), one of the MAPK family, are generally regarded as being related to cell survival, proliferation, and differentiation (Xia et al., 1995; Segal and Greenberg, 1996). Recent reports from several groups including us, however, suggested that the activation of ERK $1 / 2$ also plays crucial roles in brain inflammation including microglial activation (Zhao et al., 2007; Lu et al., 2007; Park et al., 2007).

Schizandra chinensis (S. chinensis) is widely used in traditional oriental medicine. S. chinensis extracts show hepatoprotective effect, improve eye vision in darkness, increase stamina, physical performance and working capacity, particularly in tiredness, and is recommended for treatment of patients in depressive states (Panossian and Wagner, 2005). S. chinensis has many effective components for brain function including Gomisin A, Gomisin N, Schizandrin and Schizandrol A. Gomisin A showed inhibitory effects on the activity of acetylcholinesterase enzyme (AChE) (Hung et al., 2007). Schizandrin inhibited the $\mathrm{Fe}\left({ }^{2+}\right)$-cystein-induced peroxidative damages of brain mitochondria (Xue et al., 1992). Schizandrol A exerted inhibitory effects on the central nervous system (CNS) through regulation of dopamine systems (Zhang and Niu, 1991).

Due to the essential role of neuroinflammatory processes in the progression or etiology of neurodegenerative diseases, the development of anti-neuroinflammatory agents has strong impacts on the therapeutic management of diseases including Alzheimer's disease and
Parkinson's diseases. Several compounds or mixture of compounds derived from natural products have drawn much attention in regard to the possible use as a therapeutic agent against those diseases.

Using BV2 microglial cells, which have been shown to mimic numerous primary microglial responses (Blasi et al., 1990; Bocchini et al., 1992; Murphy et al., 1998), we have investigated the effects of four compounds (Gomisin A, Gomisin N, Schizandrin and Schizandrol A) from S. chinensis on lipopolysaccharide (LPS)-induced microglial activation.

\section{MATERIALS AND METHODS}

\section{Materials}

All four S. chinensis compounds (Fig. 1) used in this study, i.e. Gomisin N, Gomisin A, Schizandrol A and Schizandrin were dissolved in $0.01 \%$ DMSO (Sigma, St. Louis, MO). These compounds were kindly supplied by the National Center for Standardization of Herbal Medicine (Seoul, Korea). Lipopolysaccharide (LPS) (026:B6) was purchased from Sigma (St. Louis, MO). Glucose-free DMEM and fetal bovine serum were from Gibco BRL (Grand Island, NY). All other reagents were obtained from Sigma (St. Louis, MO).
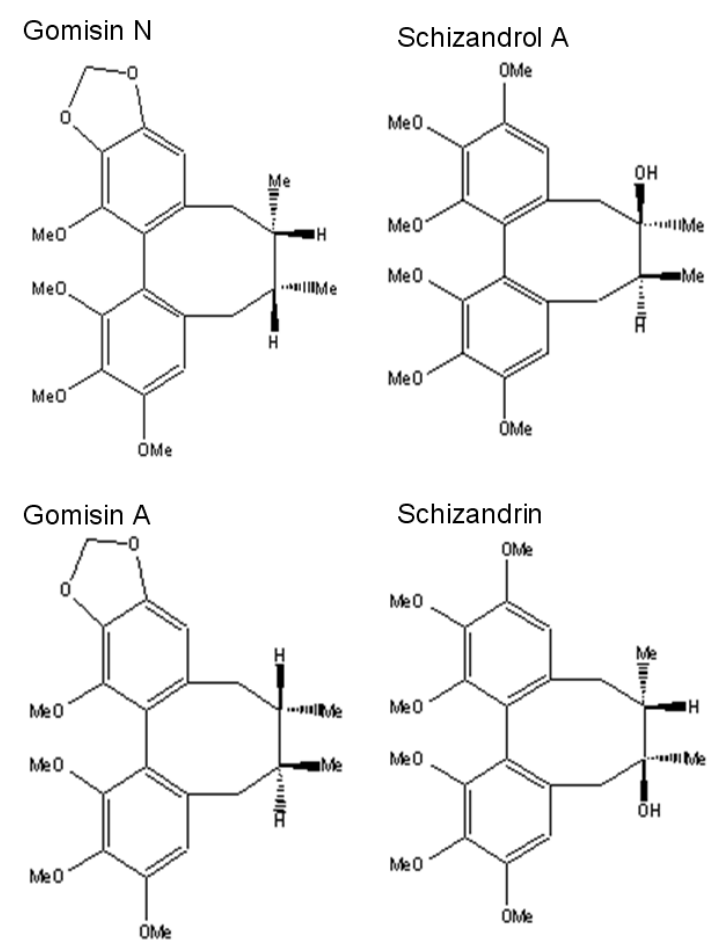

Fig. 1. Chemical structure of Gomisin A, Schizandrol A, Gomisin N and Schizandrin. 


\section{Cell culture and treatment}

The murine BV-2 cell line was maintained in DMEM supplemented with $10 \% \mathrm{FBS}, 100 \mathrm{U} / \mathrm{ml}$ penicillin and $100 \mu \mathrm{g} /$ $\mathrm{ml}$ streptomycin at $37^{\circ} \mathrm{C}$ in a humidified incubator under $5 \% \mathrm{CO}_{2}$. Confluent cultures were passed by trypsinization. For experiments, cells were washed twice with warm DMEM and then treated in serum-free medium. Cells were pretreated with S.chinensis compounds 20 min prior to LPS stimulation. After $9 \mathrm{~h}$, ROS production was determined by DCF-DA methods as described below. NO production and iNOS expression was determined $24 \mathrm{~h}$ after LPS stimulation.

\section{Measurement of ROS production}

BV2 microglial cells were loaded with 2,7-dihydrodichlorofluorescein diacetate (DCF-DA, $5 \mu \mathrm{g} / \mathrm{ml}$ ) in PBS for $10 \mathrm{~min}$ and then rinsed with the same solution. After a 30-min incubation at room temperature, the fluorescence of DCF was measured at an excitation wavelength of 485 $\mathrm{nm}$ and emission wavelength of $535 \mathrm{~nm}$ (slit widths 20 and $25 \mathrm{~nm}$, respectively) using a fluorescence microplate reader (TECAN, Austria). DCF-DA diffuses through cell membranes and is subsequently deacetylated enzymatically by intracellular esterases to the non-fluorescent DCF-H. Oxidants including $\mathrm{ONOO}^{-}$effectively convert DCF-H to the highly fluorescent DCF (Possel et al., 1997). Fluorescence intensities were corrected for autofluorescence (i.e., fluorescence of cells not loaded with DCF-DA).

\section{Determination of NO}

NO production from the immunostimulated cells was determined by measuring nitrite, a stable oxidation product of NO, as described previously (Green et al., 1990). In brief, nitrite levels were determined by adding the Griess reagent (mixing equal volumes of $0.1 \%$ napthylethylenediamine dihydrochlroride and $1 \%$ sulfanilamide in $5 \%$ phosphoric acid). After $10 \mathrm{~min}$, the absorbance at $540 \mathrm{~nm}$ was determined using an UV spectrophotometer (Beckman DU-650, Fullerton, CA).

\section{Gelatin zymography}

Zymography was performed for semi-quantitative analysis of gelatinase levels secreted into the culture medium as described by Gottschall et al. (1995), which was slightly modified by us (Lee et al., 2003). In brief, samples were mixed with $5 \mu \mathrm{l}$ of $4 \times$ SDS sample buffer ( $8 \%$ w/v SDS, $40 \%$ glycerol, $200 \mathrm{mM}$ Tris- $\mathrm{HCl}, \mathrm{pH} 6.8$, and $0.02 \%$ bromphenol blue) in the absence of a reducing agent and resolved by electrophoresis at $180 \mathrm{~V}$ for $1 \mathrm{~h}$ on $8 \%$ polyacrylamide gel containing $0.1 \%$ SDS and gelatin at a final concentration of $1 \mathrm{mg} / \mathrm{ml}$. Thereafter, gels were washed twice in $2.5 \%$ Triton $\mathrm{X}-100$ for 30 min each to remove the SDS and then incubated for $24 \mathrm{~h}$ at $37^{\circ} \mathrm{C}$ in reaction buffer (20 mM Tris- $\mathrm{HCl}, 166 \mathrm{mM} \mathrm{CaCl}_{2}$, pH 7.6). After staining the gel with $0.1 \%$ Coomassie Brilliant Blue R-250, gelatinolytic activities were visualized as a clear band in the uniformly stained background. The molecular weight of the gelatinase was estimated by comparing the migration distance of the clear bands with the distance migrated by markers of known molecular weight (Gibco BRL, Grand Island, NY).

\section{Western blot}

The activation of ERK $1 / 2$ was determined by Western blot using antibodies specific for phosphorylated, activated forms of ERK1/2. Cells were treated with LPS and/or compounds from $S$. chinensis in serum free DMEM and were lysed with $100 \mu \mathrm{l}$ of $2 \times$ sample buffer (4\% w/v SDS, $20 \%$ glycerol, $200 \mathrm{mM}$ DTT, $0.1 \mathrm{M}$ Tris- $\mathrm{HCl}, \mathrm{pH} 6.8$, and $0.02 \%$ bromophenol blue). The samples were fractionated by $10 \%$ SDS-PAGE and electrotransferred to nitrocellulose (NC) membrane. The NC membrane was blocked with $5 \%$ Blotto and then incubated at room temperature for $2 \mathrm{~h}$ with $m A b$ against $p E R K 1 / 2$ (Cell Signaling Technology, Beverly, MA, USA) which was diluted at $1: 3,000$ in $5 \%$ Blotto (Santa Cruz Biotechnology Inc., Santa Cruz, CA). After three 10 min washes with PBS containing $0.2 \%$ Tween-20 (PBS-T), the NC membranes were incubated with peroxidase-labeled goat anti-mouse $\lg G$ at room temperature for $2 \mathrm{~h}$. After extensive washing with PBS-T, the membranes were developed by enhanced chemiluminescence (Amersham, Buckinghampshire, UK). As loading controls, Western blot was performed using antibodies against $\beta$-actin in 1:30,000 dilution (Sigma).

\section{Semiquantitative RT-PCR}

Total RNA was extracted from BV2 microglial cells using Trizol reagent (GibcoBRL, Grand Island, NY). Reverse transcription was performed for $60 \mathrm{~min}$ at $45^{\circ} \mathrm{C}$ with $2 \mu \mathrm{g}$ of total RNA using Maxime RT PreMix Kit (iNtRON Biotechnology, Seoul) according to the manufacturer's protocol. Oligo $(\mathrm{dT})_{15}$ was used as a primer for this reaction. The samples were then heated at $94^{\circ} \mathrm{C}$ for $5 \mathrm{~min}$ to terminate the reaction. The cDNA obtained from $0.5 \mu \mathrm{g}$ total RNA was used as a template for PCR amplification of MMP-9, iNOS (accession number U03699) and glyceraldehyde 3-phosphate dehydrogenase (GAPDH, accession number M17701) mRNA as described previously (Lee et al., 2003). The following Primers were used for amplification reaction: for MMP9, 
forward primer 5'-TAAGGTATTCAGTTACTCCTACTGGAA-3'

reverse primer 5'-CCTCTCTAGCACACATGCACTT-3' for iNOS,

forward primer 5'-CAAGAGTTTGACCAGAGGACC-3' reverse primer 5'-TGGAACCACTCGTACTTGGGA-3' for GAPDH,

forward primer 5'-TCCCTCAAGATTGTCAGCAA-3' reverse primer 5'-AGATCCACAACGGATACATT-3'

PCR mixture contained $1 \mathrm{pmol}$ of each forward and reverse primer and $5 \mu \mathrm{l}$ of each cDNA and Maxime PCR PreMix Kit (i-Taq). For amplification, the following PCR incubation times were used: $94^{\circ} \mathrm{C}$ for $5 \mathrm{~min}$ followed by 28 cycles of $94^{\circ} \mathrm{C}$ for $30 \mathrm{~s}, 55^{\circ} \mathrm{C}$ for $30 \mathrm{~s}, 72^{\circ} \mathrm{C}$ for $1 \mathrm{~min}$ and continued by an final extension step at $72^{\circ} \mathrm{C}$ for $10 \mathrm{~min}$. The amplified PCR products were electrophoresed and analysed on $1 \%$ agarose-gel. For comparison, PCR reaction for housekeeping gene, GAPDH, was also performed. The expected size of the amplified DNA fragments was 654 bp for iNOS, 324 bp for MMP9, and 308 bp for GAPDH .

\section{Transient transfection and MMP-9 promoter reporter assay}

BV2 microglial cells were co-transfected with MMP-9 promoter-luciferase reporter plasmid and pCMV- $\beta$-galactosidase reporter plasmid using Lipofectamine 2000 reagent as reported previously (Kim et al., 2007). Next day, BV2 microglial cells were pretreated with Schizandra chinensis compounds $(20 \mu \mathrm{M})$ and then treated with LPS $(0.2$ $\mu \mathrm{g} / \mathrm{ml})$ in serum-free DMEM condition. Luciferase and $\beta$-galactosidase activities were assayed by using the luciferase and $\beta$-galactosidase enzyme assay system (Promega, Madison, $\mathrm{WI}$ ), respectively. Luciferase activity was normalized with the $\beta$-galactosidase activity in the cell lysates.

\section{Statistical analysis}

Data are expressed as the mean \pm standard deviation (S.D.) and analyzed for statistical significance by using one way analysis of variance (ANOVA) followed by Student's $t$-test. Data with values of $p<0.05$ were generally accepted as statistically significant.

\section{RESULTS}

\section{S. chinensis compounds reduced ROS generation in LPS-stimulated microglia}

The chemical structure of four compounds used in this study was depicted in Fig. 1. To investigate whether S. chinensis compounds affect ROS generation, BV2 microglial cells were pre-treated with each $S$. chinensis compounds $(20 \mu \mathrm{M})$ and immunostimulated with LPS $(0.2 \mu \mathrm{g} / \mathrm{ml})$ for 9 hrs. The concentration $(20 \mu \mathrm{M})$ was pre-determined, which did not cause a significant effect on cell viability that was determined by MTT reduction assay (data not shown).
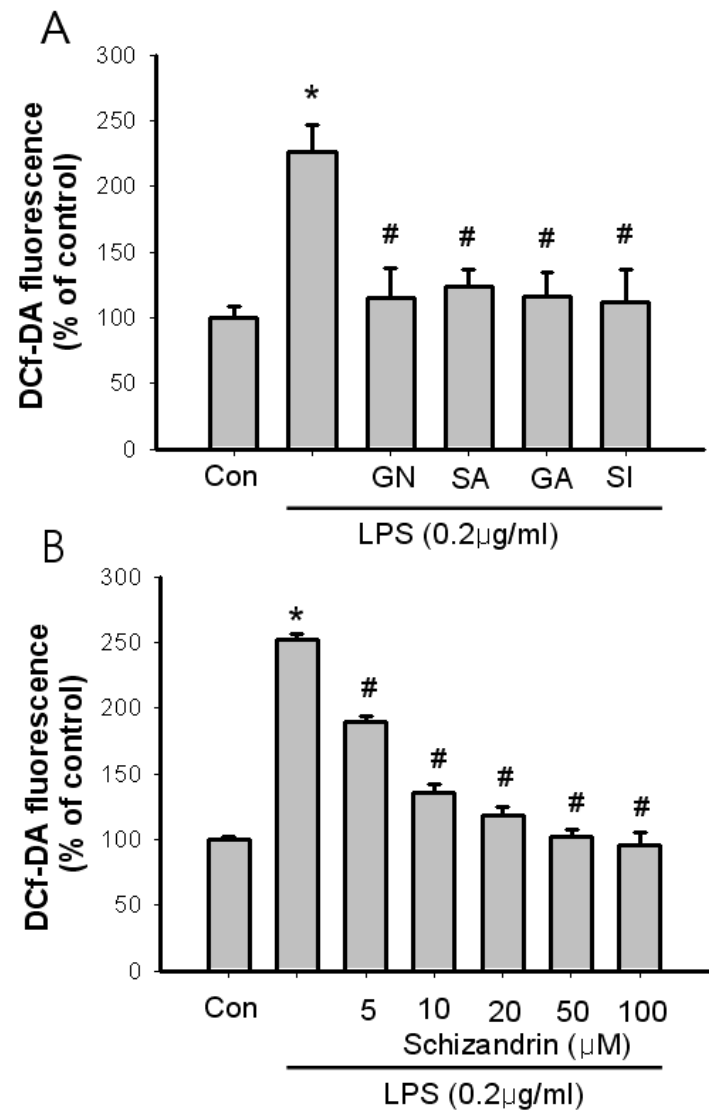

Fig. 2. Inhibitory effects of $S$. chinensis compounds on ROS generation in BV2 microglial cells. BV2 cells were pretreated with each $20 \mu \mathrm{M}$ compounds from S. chinensis (A) or different concentration of Schizandrin (B) followed by $0.2 \mu \mathrm{g} / \mathrm{ml}$ LPS treatment. Then, cells were incubated with 2,7-dihydrodichlorofluorescein diacetate for $30 \mathrm{~min}$. The fluorescence of DCF was measured at an excitation wavelength of $485 \mathrm{~nm}$ and emission wavelength of $535 \mathrm{~nm}$ using a fluorescence microplate reader. Results are the mean \pm standard deviation (S.D.) of three independent experiments. Asterisk $\left({ }^{*}\right)$ indicates significant induction compared with non-treated control. \# indicates significant difference from LPS-stimulated group ( $p$ $<0.05)$. 
BV2 cells exposed to LPS showed a significant increase (approximately two-fold) in intracellular ROS level, and this increase was returned to near basal level by treatment with S. chinensis compounds, i.e. Gomisin A, Gomisin N, Schizandrin and Schizandrol A (Fig. 2A). The concentration-response relationship of Schizandrin (Fig. 2B) showed that even at $5 \mu \mathrm{M}$ concentration, Schizandrin significantly inhibited LPS-induced increase in ROS production in rat primary astrocytes.

\section{S. chinensis compounds inhibited LPS-induced NO production}

Next, we determined the level of NO, which plays important roles in the modulation of neuroinflammatory processes in the brain. NO production was assayed by measuring the levels of a stable NO metabolite, nitrite in the conditioned medium. All four compounds of $S$. chinensis decreased LPS-induced NO production (Fig. 3A). Gomisin A, Gomisin N, Schizandrin and Schizandrol A effectively inhibited NO production by $20-40 \%$. The potency of inhibition was in the order of Gomisin $\mathrm{N}<$ Schizandrol $\mathrm{A}<$ Gomisin A $<$ Schizandrin.

\section{Inhibition of iNOS expression by S. chinensis}

To determine whether the inhibitory effect of $S$. chinensis compounds on NO production was due to the decrease in the cytosolic iNOS protein level, BV-2 microglial cells were treated with $S$. chinensis compounds and/or with LPS, and the levels of iNOS protein and MRNA were detected by Western blot and RT-PCR, respectively. As shown in Fig. 3, all four compounds of $S$. chinensis decreased LPS-induced iNOS mRNA (Fig. 3B) and protein expression (Fig. $3 \mathrm{C}$ ) suggesting that the inhibition of LPS-induced NO production by S. chinensis was mediated by the inhibition of iNOS expression.

The extent of inhibition was relatively weak with Gomisin $\mathrm{N}$ and there was no statistical difference in mRNA and protein level albeit there was a tendency of decreased expression by Gomisin N. Likewise, the level of mRNA was not significantly different with Schizandrol $A$ although there was a clear tendency of inhibition of iNOS mRNA expression by Schizandrol A (Fig. 3B).

\section{S. chinensis compounds inhibited LPS-induced MMP-9 expression}

In addition to ROS and RNS, proteases including MMPs

\section{A. NO Assay}
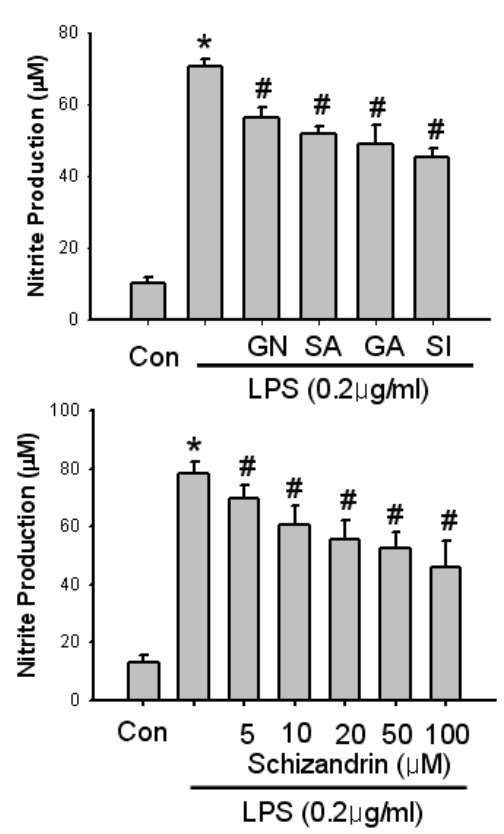

B. iNOS RT-PCR

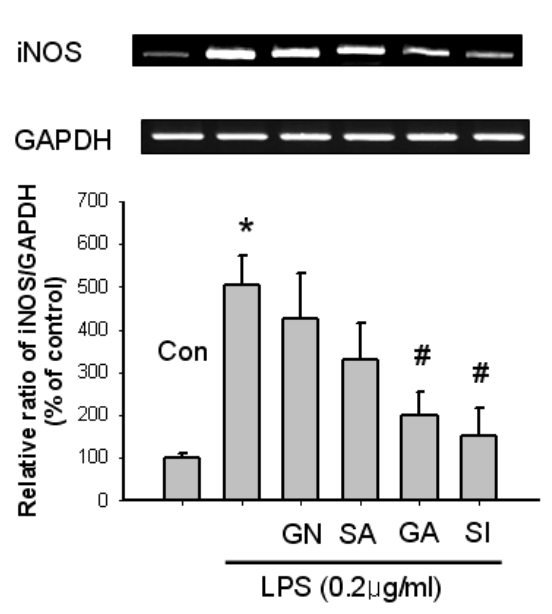

iNOS

$\beta$-Actin

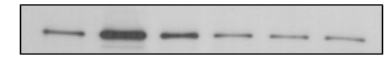

(2.

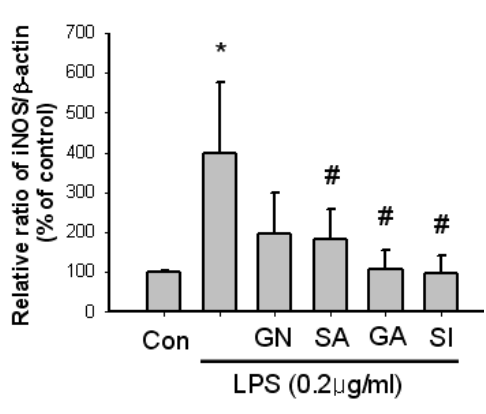

Fig. 3. S. chinensis compounds reduced NO production by the inhibition of iNOS expression. BV2 cells were pretreated with each 20 $\mu \mathrm{M}$ compounds from S. chinensis or different concentrations of Schizandrin followed by $0.2 \mu \mathrm{g} / \mathrm{ml}$ LPS treatment. (A) Cultured medium was collected, and nitrite level was determined by Griess reaction. (B) Treated cells were collected, and RT-PCR assays against iNOS mRNA were performed. (C) Western blot for iNOS. Lower panel shows quantitative data. Results are the mean \pm standard deviation (S.D.) of three independent experiments. Asterisk $\left(^{*}\right)$ indicates significant induction compared with non-treated control. \# indicates significant difference from LPS-stimulated group $(p<0.05)$. 
and TPA is induced after immunological insult condition in the brain, which regulates many different neuropathological processes including extracellular matrix digestion, regulation of cell migration and regeneration. To inves-

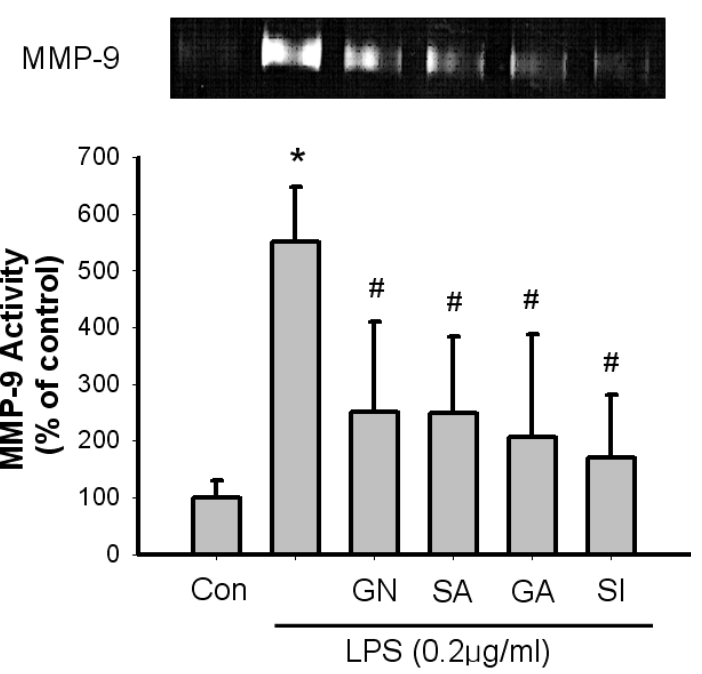

Fig. 4. MMP-9 Activity was reduced by all four S. chinensis compounds. BV2 cells were pretreated with each $20 \mu \mathrm{M}$ compounds of $S$. chinensis followed by $0.2 \mu \mathrm{g} / \mathrm{ml}$ LPS treatment. Then, conditioned medium was collected and zymography was performed as indicated in materials and methods. Upper panel shows representative figure, and lower panel shows quantitative data. Results are the mean \pm standard deviation (S.D.) of three independent experiments. Asterisk ( $\left.{ }^{*}\right)$ indicates significant induction compared with non-treated control. \# indicates significant difference from LPS-stimulated group $(p<0.05)$. tigate the effect of S. chinensis compounds on MMP-9 expression, BV2 microglial cells were treated with $S$. chinensis compounds and/or with LPS. The activity of MMP-9 and MMP-2 was determined by gelatin zymography. LPS induced the expression of MMP-9 and pre-treatment with four S. chinensis compounds decreased LPS-induced MMP-9 expression (Fig. 4, 5). LPS treatment did not affect MMP-2 activity as we reported previously (data not shown, Lee et al., 2003). The potency of the inhibition was in the order of Gomisin $\mathrm{N}<$ Schizandrol $\mathrm{A}<$ Gomisin $\mathrm{A}<$ Schizandrin, which is similar to the NO inhibition experiments (Fig. 3A). RT-PCR was also performed to determine the effect of $S$. chinensis compounds on MMP-9 mRNA level. Fig. 5B showed that pre-treatment with each of the four $S$. chinensis compounds decreased the LPS-induced MMP-9 mRNA expression suggesting that the regulation of MMP-9 activity by S. chinensis compounds is mediated by transcriptional control of MMP-9 gene expression. The regulation of MMP-9 gene transcription was further confirmed by MMP-9 promoter-luciferase activity assay. As shown in Fig. 5A, LPS stimulated MMP-9 promoter-luciferase activity and pre-treatment of each of the four $S$. chinensis compounds decreased LPS-induced MMP-9 promoter luciferase activity to control level.

\section{Inhibition of ERK1/2 phosphorylation by S. chinensis}

Since production of numerous inflammatory mediators including NO and MMP-9 were ERK1/2 MAPK dependent in glial cells, we investigated whether $S$. chinensis compounds inhibit ERK1/2 activation. Fig. 6 showed that 30 min incubation with $0.2 \mu \mathrm{g} / \mathrm{ml}$ LPS activated ERK1/2,

\section{$\begin{array}{ll}\text { A. Reporter Assay } & \text { B. RT-PCR }\end{array}$}

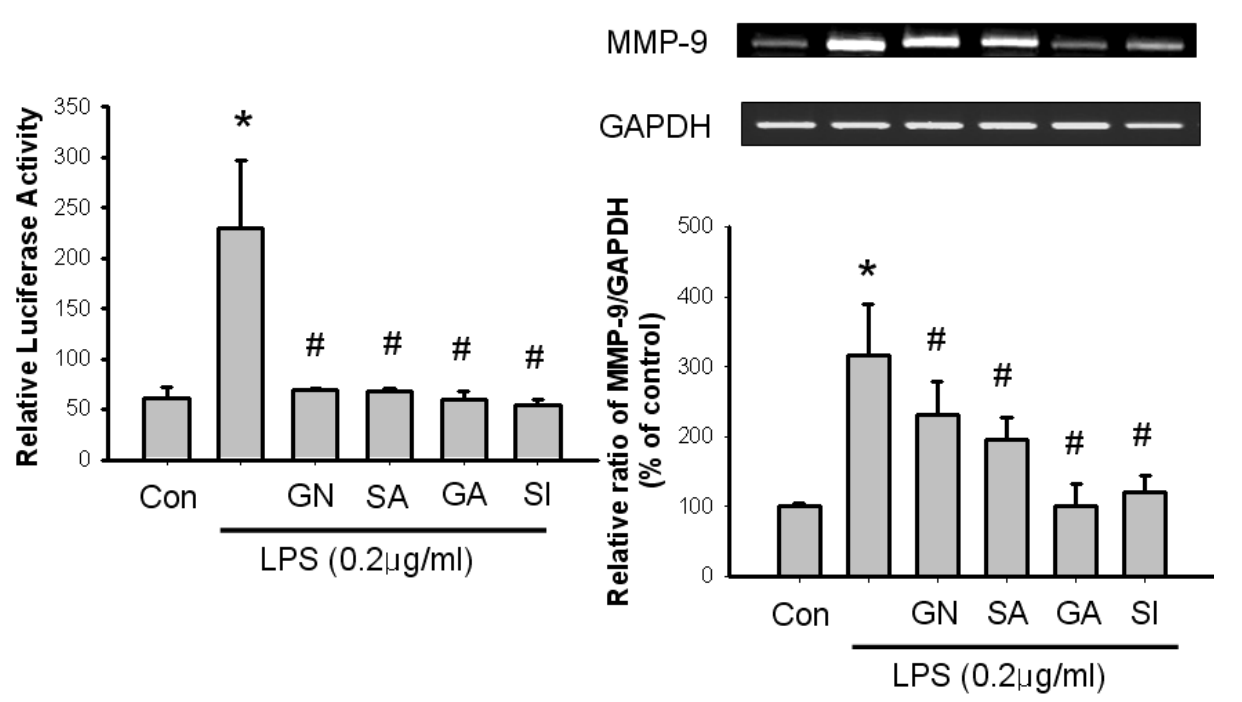

Fig. 5. Inhibition of MMP-9 expression by $S$. chinensis compounds. BV2 cells were pretreated with each $20 \mu \mathrm{M}$ compounds from S. chinensis followed by $0.2 \mu \mathrm{g} / \mathrm{ml}$ LPS treatment. (A) MMP-9 reporter assay was carried out using Promega kit as described in materials and methods. (B) MMP-9 mRNA level was detected using RT-PCR. Results are the mean \pm standard deviation (S.D.) of three independent experiments. Asterisk $\left(^{*}\right)$ indicates significant induction compared with non-treated control. \# indicates significant difference from LPS-stimulated group $(p<0.05)$. 


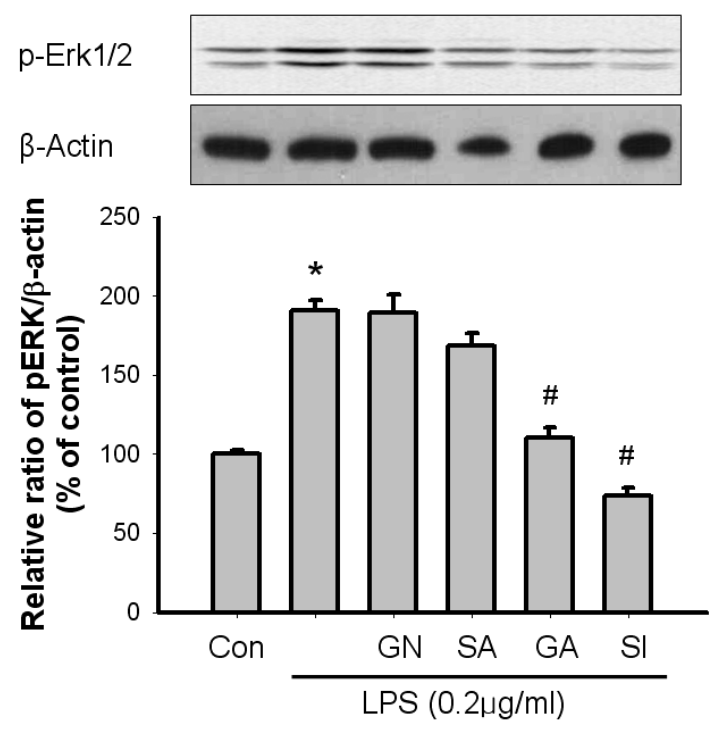

Fig. 6. Effects of $S$. chinensis compounds on LPS-induced phosphorylation of ERK1/2. BV2 cells were pretreated with each $20 \mu \mathrm{M}$ compounds from $S$. chinensis followed by 0.2 $\mu \mathrm{g} / \mathrm{ml}$ LPS treatment. After $2 \mathrm{~h}$, Western blot against pERK1/2 were performed. Results are the mean \pm standard deviation (S.D.) of three independent experiments. Asterisk $\left({ }^{*}\right)$ indicates significant difference compared with non-treated control. \# indicates significant difference from LPS-stimulated group $(p<$ 0.05).

which is consistent with previous reports (Lee et al., 2003) and the increased activity was significantly suppressed by pre-incubation with $S$. chinensis compounds $(20 \mu \mathrm{M}$, respectively) for $20 \mathrm{~min}$. Again, the potency of inhibition of ERK1/2 phosphorylation was in the order of Gomisin N < Schizandrol $A<$ Gomisin $A<$ Schizandrin. Gomisin $N$ and Schizandrol A tend to inhibit ERK1/2 phosphorylation although there is no statistical significance. The weak inhibitory effects of Gomisin N and Schizandrol A on ERK1/2 phosphorylation were consistent with the weak inhibitory effects of these compounds on NO production as well. There was no change in the level of total ERK2 (data not shown) or $\beta$-actin (Fig. 6) as determined by Western blot using antibodies specific against total ERK2 or $\beta$-actin.

\section{DISCUSSION}

In this report, we demonstrated that four compounds (Gomisin A, Gomisin N, Schizandrin and Schizandrol A) from $S$. chinensis suppressed microglial activation. All four compounds from $S$. chinensis significantly decreased LPS-induced production of inflammatory mediators such as ROS (Fig. 2), NO (Fig. 3), iNOS (Fig. 3) and MMP-9 (Fig. 4,5$)$ as well as the activation of ERK1/2 (Fig. 6) in
LPS-stimulated BV2 microglial cells. These compounds can be classified as lignans and have two methylated phenol groups in their core structure, which is a recurrent structural feature in many polyphenol compounds with anti-oxidant activity. In general, Schizandrin or Schizandrol A has stronger anti-inflammatory activity compared with Gomisin N suggesting that the addition of dioxolane ring structure diminishes the anti-inflammatory activity. However, care should be given not to over-simplify the anti-inflammatory structural features of these compounds, which needs detailed study and interpretation in the future.

We used LPS to immunologically stimulate BV2 microglial cells. Many researchers including us used LPS stimulation as a general model system for immunological stimulation of glial cells including microglia and astrocytes. LPS-stimulation induced strong and consistent immunological activation of glial cells through its action on TLR4, which might be related to the inflammatory burden of neurodegenerative diseases such as Alzheimer's disease.

Although microglial activation is one of the body's normal responses against brain injury, inflammatory mediators released from activated microglia may induce neuronal injury, leading to neurodegenerative diseases. It has been reported that toxic molecules released by activated neuroglial cells might be involved in the pathogenesis of various neurodegenerative diseases such as Parkinson's disease (PD), Alzheimer's disease (AD) and HIV-associated dementia (Gonzalez-Scarano and Baltuch, 1999; McGeer and McGeer, 1995; Minghetti and Levi, 1998). Thus, many researchers have focused on the use of natural products to control the production of neuroinflammatory regulators, especially from microglia (Fiebich et al., 2005; Jung et al., 2007; Lau et al., 2007). The present study demonstrated that some compounds from $S$. chinensis suppressed microglial activation. Although $S$. chinensis has many effective compounds regulating brain function, there has been no report regarding the possible anti-neuroinflammatory action. In one study, anti-inflammatory activity of Gomisin A was reported (Yasukawa et al., 1992), but in this case, it was performed in the model of TPA-induced skin tumor formation in mice, and the other two studies were performed in the hepatotoxicity model (Ohkura et al., 1990; Yamada et al., 1993).

Excessive ROS production may induce inflammatory response and it has been reported that antioxidants inhibit NF- $\mathrm{KB}$ activation and suppress the expression of inflammatory cytokines including NO (Tsai et al., 1999; Liang et al., 1999). Additionally, Lin et al. reported that over-expression of catalase attenuates ROS production and subsequently inactivates AP-1 in endothelial cells (Lin 
et al., 2004). The strong antioxidant effects of $S$. chinensis compounds may, at least in part, explain the anti-inflammatory effects in microglial cells.

In the present study, S. chinensis compounds inhibited LPS-induced iNOS activity and NO production (Fig. 3) in BV2 microglial cells. It has been reported that NO modulates COX-2 activity in a cGMP-independent manner, and NO plays a critical role in the release of PGE2 by the direct activation of COX-2 (Salvemini et al., 1993). Considering the essential role of COX-2 in inflammatory cascade, investigating the role of $S$. chinensis compounds on COX-2 activity in BV2 microglial cells may provide further evidence of the anti-inflammatory action of these compounds. Consistent with the above notion, Schizandrin has been reported to reduce LPS-induced COX-2 expression in RAW 264.7 macrophageal cell line (Guo et al., 2008).

Additionally, exposure to endogenous and exogenous stimulators including LPS induced iNOS and triggered deleterious cellular responses inducing inflammation, sepsis, and stroke (Nakashima et al., 2003; Semmler et al., 2005). Therefore, the inhibitory effect of $S$. chinensis for $\mathrm{NO}$ and iNOS can have wide-spreading effects in the regulation of neuroinflammatory response of LPS-stimulated brain as in the case of modulation of ROS production.

In this study, the extent of inhibition of NO production (Fig. 3A) was less prominent compared with that of iNOS protein expression (Fig. 3C). The differential regulation of other subtypes of NOS such as eNOS or delayed inhibition of iNOS protein expression compared to NO accumulation may provide additional insights into the exact mechanism of NO regulation by S. chinensis compounds, which should be investigated further in the future study.

In this study, LPS-induced NO production as well as iNOS expression was inhibited by $S$. chinensis compounds. However, constitutive NO production was not inhibited by these compounds (data not shown) suggesting that NO inhibition is not mediated by the diirect inhibition of NOS activity.

Increased MMP-9 activity has been reported in the cerebrospinal fluid (CSF) of MS patients, and this protease activity has generally been thought to be reflective of the inflammatory process (Gijbels et al., 1994; Kieseier et al., 1999). Increased level of MMP-9 release from microglia may worsen the severity of neuroinflammatory diseases. It can be postulated that the inhibitory effect of $S$. chinensis against MMP-9 production (Fig. 4, 5), as found in this study, may provide anti-inflammatory effect in brain, which might be beneficial in several neuropathological conditions including MS. However, it has been also suggested that some MMPs, upregulated following CNS injury, may con- tribute to the recovery of the CNS function (Yong et al., 1998). Care should be given to determine whether the inhibitory effect of $S$. chinensis for MMP-9 production would be beneficial or detrimental. In this regard, determining the time window as well as the localization of MMP-9 induction after brain injury may have profound impact in devising best way to prevent neural damage in neuroinflammatory condition. The use of $S$. chinensis compounds may have beneficial effects in brain injury by modulating MMP-9 induction.

Activation of ERK1/2 plays crucial role in the regulation of inflammatory phenotype, not only in iNOS and MMP induction but also in other parameters of inflammatory responses. For example, Zhao et al. showed that PGE2 release was dependent on microglial activation and ERK1/2 phosphorylation, and also showed COX-2 expression in microglia was reduced by MEK1/2 inhibition (Zhao et al., 2007). Considering the inhibitory role of $S$. chinensis for ERK1/2 activation (Fig. 6), it would be also interesting to examine whether $S$. chinensis compounds inhibit LPS-induced PGE2 and/or COX-2 in microglial cells.

In summary, for the first time, we showed that four compounds (Gomisin A, Gomisin N, Schizandrin and Schizandrol A) from $S$. chinensis suppressed microglial activation through the inhibition of the intracellular level of ROS, the release of NO and MMP-9, the phosphorylation of ERK1/2 which were induced by LPS. Because it is almost without doubt that the inhibition of microglial activation is a critical target for regulation of neuroinflammatory phenotype in brain, the results from the present study suggest that the four compounds from S. chinensis play a protective role in the neuroinflammatory condition by inhibiting the production of toxic inflammatory mediators in microglia. S. chinensis has anticholinesterase activity (Hung et al., 2007), which may be helpful in ameliorating the memory deficits in Alzheimer's disease patients. Whether anti-inflammatory effects of natural compounds from S. chinesis contribute to the recovery of functional outcome in brain insults condition, is an open question we eager to answer in the near future.

\section{ACKNOWLEDGMENTS}

This research was supported by a grant (M103KV010025-06K2201-02510) from Brain Research Center of the $21^{\text {st }}$ Century Frontier Research Program funded by the Ministry of Science and Technology, the Republic of Korea, and by a grant (L-05-03-2-CHM-628-B) funded by the Korean Food and Drug Administration. 


\section{REFERENCES}

Banati, R. B., Daniel, S. E. and Blunt, S. B. (1998). Glial pathology but absence of apoptotic nigral neurons in long-standing Parkinson's disease. Mov. Disord. 13, 221227.

Beckman, J. S., Beckman, T. W., Chen, J., Marshall, P. A. and Freeman, B. A. (1990). Apparent hydroxyl radical production by peroxynitrite: implications for endothelial injury from nitric oxide and superoxide. Proc Natl Acad Sci U S A 87, 1620-1624.

Blasi, E., Barluzzi, R., Bocchini, V., Mazzolla, R. and Bistoni, F. (1990). Immortalization of murine microglial cells by a v-raf/v-myc carrying retrovirus. J. Neuroimmunol. 27, 229237.

Bocchini, V., Mazzolla, R., Barluzzi, R., Blasi, E., Sick, P. and Kettenmann, H. (1992). An immortalized cell line expresses properties of activated microglial cells. J. Neurosci. Res. 31, 616-621.

Bornemann, K.D., Wiederhold, K.H., Pauli, C., Ermini, F., Stalder, M., Schnell, L., Sommer, B., Jucker, M. and Staufenbiel, M. (2001). Abeta-induced inflammatory processes in microglia cells of APP23 transgenic mice. Am. J. Pathol. 158, 63-73.

Bruhwyler, J., Chleide, E., Liegeois, J.F. and Carreer, F. (1993). Nitric oxide: a new messenger in the brain. Neurosci. Biobehav. Rev. 17, 373-384.

Chang, L. and Karin, M. (2001). Mammalian MAP kinase signalling cascades. Nature. 410, 37-40.

Choi, J. J. and Kim, W. K. (1998). Potentiated glucose deprivation-induced death of astrocytes after induction of iNOS. J. Neurosci. Res. 54, 870-875.

Cuzner, M. L. and Opdenakker, G. (1999). Plasminogen activators and matrix metalloproteases, mediators of extracellular proteolysis in inflammatory demyelination of the central nervous system. J. Neuroimmunol. 94, 1-14.

Darley-Usmar, V., Wiseman, H. and Halliwell, B. (1995). Nitric oxide and oxygen radicals: a question of balance. FEBS. Lett. 369, 131-135.

del Zoppo, G. J., Milner, R., Mabuchi, T., Hung, S., Wang, X., Berg, G. I. and Koziol, J. A. (2007). Microglial activation and matrix protease generation during focal cerebral ischemia. Stroke. 38, 646-651.

Fiebich, B. L., Grozdeva, M., Hess, S., Hull, M., Danesch, U., Bodensieck, A. and Bauer, R. (2005). Petasites hybridus extracts in vitro inhibit COX-2 and PGE2 release by direct interaction with the enzyme and by preventing p42/44 MAP kinase activation in rat primary microglial cells. Planta. Med. 71, 12-19.

Gijbels, K., Galardy, R. E. and Steinman, L. (1994). Reversal of experimental autoimmune encephalomyelitis with a hydroxamate inhibitor of matrix metalloproteases. J. Clin. Invest. 94, 2177-2182.

Gloire, G., Legrand-Poels, S. and Piette, J. (2006). NF-kappaB activation by reactive oxygen species: fifteen years later. Biochem. Pharmacol. 72, 1493-1505

Gonzalez-Scarano, F. and Baltuch, G. (1999). Microglia as mediators of inflammatory and degenerative diseases. Annu. Rev. Neurosci. 22, 219-240.
Gottschall, P. E., Yu, X. and Bing, B. (1995). Increased production of gelatinase $B$ (matrix metalloproteinase-9) and interleukin- 6 by activated rat microglia in culture. J. Neurosci. Res. 42, 335-342.

Green, T. R. and Pratt, K. L. (1990). Detection and isolation of the NADPH-binding protein of the NADPH:O2 oxidoreductase complex of human neutrophils. J. Biol. Chem. 265, 19324-19329.

Guo, L. Y., Hung, T. M., Bae, K. H., Shin, E. M., Zhou, H. Y., Hong, Y. N., Kang, S. S., Kim, H. P. and Kim, Y. S. (2008) Anti-inflammatory effects of schisandrin isolated from the fruit of Schisandra chinensis Baill. Eur. J. Pharmacol. 591, 293-299.

Hensley, K., Hall, N., Subramaniam, R., Cole, P., Harris, M., Aksenov, M., Aksenova, M., Gabbita, S. P., Wu, J. F., Carney, J. M., Lovell, M., William., Markesbery R., Allan Butterfield, D. (1995). Brain regional correspondence between Alzheimer's disease histopathology and biomarkers of protein oxidation. J. Neurochem. 65, 2146-2156.

Hewett, S. J., Csernansky, C. A. and Choi, D. W. (1994). Selective potentiation of NMDA-induced neuronal injury following induction of astrocytic iNOS. Neuron. 13, 487-494.

Hung, T. M., Na, M., Min, B. S., Ngoc, T. M., Lee, I., Zhang, X. and Bae, K. (2007). Acetylcholinesterase inhibitory effect of lignans isolated from Schizandra chinensis. Arch. Pharm. Res. 30, 685-690.

Jung, K., Ha, E., Uhm, Y., Park, H., Kim, M.J., Kim, H., Baik, H., Hong, M., Yang, J. and Yim, S. V. (2007). Suppressive effect by Hizikia fusiforme on the production of tumor necrosis factor in BV2 murine microglial cells. Neurol. Res. 29, 88-92.

Kieseier, B.C., Seifert, T., Giovannoni, G. and Hartung, H.P. (1999). Matrix metalloproteinases in inflammatory demyelination: targets for treatment. Neurology. 53, 20-25.

Kim, S. D., Yang, S. I., Kim, H. C., Shin, C. Y. and Ko, K. H. (2007). Inhibition of GSK-3beta mediates expression of MMP-9 through ERK1/2 activation and translocation of NF-kappaB in rat primary astrocyte. Brain. Res. 1186, 12-20.

Lau, F. C., Bielinski, D. F. and Joseph, J. A. (2007). Inhibitory effects of blueberry extract on the production of inflammatory mediators in lipopolysaccharide-activated BV2 microglia. $J$. Neurosci. Res. 85, 1010-1017.

Lee, W. J., Shin, C. Y., Yoo, B. K., Ryu, J. R., Choi, E. Y., Cheong, J. H., Ryu, J. H. and Ko, K. H. (2003). Induction of matrix metalloproteinase-9 (MMP-9) in lipopolysaccharidestimulated primary astrocytes is mediated by extracellular signal-regulated protein kinase 1/2 (Erk1/2). Glia. 41, 15-24.

Liang, Y. C., Huang, Y. T., Tsai, S. H., Lin-Shiau, S. Y., Chen, C. F. and Lin, J. K. (1999). Suppression of inducible cyclooxygenase and inducible nitric oxide synthase by apigenin and related flavonoids in mouse macrophages. Carcinogenesis. 20, 1945-1952.

Lin, S. J., Shyue, S. K., Liu, P. L., Chen, Y. H., Ku, H. H., Chen, J. W., Tam, K. B. and Chen, Y. L. (2004). Adenovirus-mediated overexpression of catalase attenuates oxLDL-induced apoptosis in human aortic endothelial cells via AP-1 and C-Jun N-terminal kinase/extracellular signal-regulated kinase mitogen-activated protein kinase pathways. J. Mol. Cell. Cardiol. 36, 129-139.

Lu, K., Cho, C. L., Liang, C. L., Chen, S. D., Liliang, P. C., Wang, S. Y. and Chen, H. J. (2007). Inhibition of the MEK/ERK pathway reduces microglial activation and 
interleukin-1-beta expression in spinal cord ischemia/ reperfusion injury in rats. J. Thorac. Cardiovasc. Surg. 133, 934-941.

McGeer, P. L. and McGeer, E. G. (1995). The inflammatory response system of brain: implications for therapy of Alzheimer and other neurodegenerative diseases. Brain. Res. Brain. Res. Rev. 21, 195-218.

McGeer, P. L. and McGeer, E. G. (2004a). Inflammation and neurodegeneration in Parkinson's disease. Parkinsonism. Relat. Disord. 10, 3-7.

McGeer, P. L. and McGeer, E. G. (2004b). Inflammation and the degenerative diseases of aging. Ann. N. Y. Acad. Sci. 1035, 104-116.

Minghetti, L. and Levi, G. (1998). Microglia as effector cells in brain damage and repair: focus on prostanoids and nitric oxide. Prog. Neurobiol. 54, 99-125.

Murphy, G. M., Jr., Yang, L. and Cordell, B. (1998). Macrophage colony-stimulating factor augments beta-amyloid-induced interleukin-1, interleukin-6, and nitric oxide production by microglial cells. J. Biol. Chem. 273, 20967-20971.

Nakashima, M. N., Ajiki, K., Nakashima, K. and Takahashi, M. (2003). Possible role of nitric oxide in anxiety following transient cerebral ischemia in mice. J. Pharmacol. Sci. 91, 47-52.

Ohkura, Y., Mizoguchi, Y., Morisawa, S., Takeda, S., Aburada, M. and Hosoya, E. (1990). Effect of gomisin A (TJN-101) on the arachidonic acid cascade in macrophages. Jpn. J. Pharmacol. 52, 331-336.

Panossian, A. and Wagner, H. (2005). Stimulating effect of adaptogens: an overview with particular reference to their efficacy following single dose administration. Phytother. Res. 19, 819-838.

Park, J. S., Woo, M. S., Kim, D. H., Hyun, J. W., Kim, W. K., Lee, J. C. and Kim, H. S. (2007). Anti-inflammatory mechanisms of isoflavone metabolites in lipopolysaccharide-stimulated microglial cells. J. Pharmacol. Exp. Ther. 320, 1237-1245.

Pham, C. G., Bubici, C., Zazzeroni, F., Papa, S., Jones, J., Alvarez, K., Jayawardena, S., De Smaele, E., Cong, R. Beaumont, C., Torti, F. M., Torti, S. V. and Franzoso, G. (2004). Ferritin heavy chain upregulation by NF-kappaB inhibits TNFalpha-induced apoptosis by suppressing reactive oxygen species. Cell. 119, 529-542.

Possel, H., Noack, H., Augustin, W., Keilhoff, G. and Wolf, G. (1997). 2,7-Dihydrodichlorofluorescein diacetate as a fluorescent marker for peroxynitrite formation. FEBS. Lett. $\mathbf{4 1 6}$
$175-178$

Salvemini, D., Misko, T. P., Masferrer, J. L., Seibert, K., Currie, M. G. and Needleman, P. (1993). Nitric oxide activates cyclooxygenase enzymes. Proc. Natl. Acad. Sci. U S A. 90, 7240-7244.

Segal, R. A. and Greenberg, M. E. (1996). Intracellular signaling pathways activated by neurotrophic factors. Annu. Rev. Neurosci. 19, 463-489.

Semmler, A., Okulla, T., Sastre, M., Dumitrescu-Ozimek, L. and Heneka, M. T. (2005). Systemic inflammation induces apoptosis with variable vulnerability of different brain regions. J. Chem. Neuroanat. 30, 144-157.

Tsai, S. H., Lin-Shiau, S. Y. and Lin, J. K. (1999). Suppression of nitric oxide synthase and the down-regulation of the activation of NFkappaB in macrophages by resveratrol. Br. J. Pharmacol. 126, 673-680.

Xia, Z., Dickens, M., Raingeaud, J., Davis, R. J. and Greenberg, M.E. (1995). Opposing effects of ERK and JNK-p38 MAP kinases on apoptosis. Science. 270, 1326-1331.

Xue, J. Y., Liu, G. T., Wei, H. L. and Pan, Y. (1992). Antioxidant activity of two dibenzocyclooctene lignans on the aged and ischemic brain in rats. Free. Radic. Biol. Med. 12, 127-135.

Yamada, S., Murawaki, Y. and Kawasaki, H. (1993). Preventive effect of gomisin A, a lignan component of shizandra fruits, on acetaminophen-induced hepatotoxicity in rats. Biochem. Pharmacol. 46, 1081-1085.

Yasukawa, K., Ikeya, Y., Mitsuhashi, H., Iwasaki, M., Aburada, M., Nakagawa, S., Takeuchi, M. and Takido, M. (1992). Gomisin A inhibits tumor promotion by 12-O-tetradecanoylphorbol-13-acetate in two-stage carcinogenesis in mouse skin. Oncology. 49, 68-71.

Yong, V. W., Krekoski, C. A., Forsyth, P. A., Bell, R. and Edwards, D. R. (1998). Matrix metalloproteinases and diseases of the CNS. Trends. Neurosci. 21, 75-80.

Yu, A. E., Hewitt, R. E., Connor, E. W. and Stetler-Stevenson, W. G. (1997). Matrix metalloproteinases. Novel targets for directed cancer therapy. Drugs. Aging. 11, 229-244.

Zhang, L. and Niu, X. (1991). Effects of schizandrol A on monoamine neurotransmitters in the central nervous system. Zhongguo. Yi. Xue. Ke. Xue. Yuan. Xue. Bao. 13, 13-16.

Zhao, P., Waxman, S. G. and Hains, B. C. (2007). Extracellular signal-regulated kinase-regulated microglia-neuron signaling by prostaglandin E2 contributes to pain after spinal cord injury. J. Neurosci. 27, 2357-2368. 